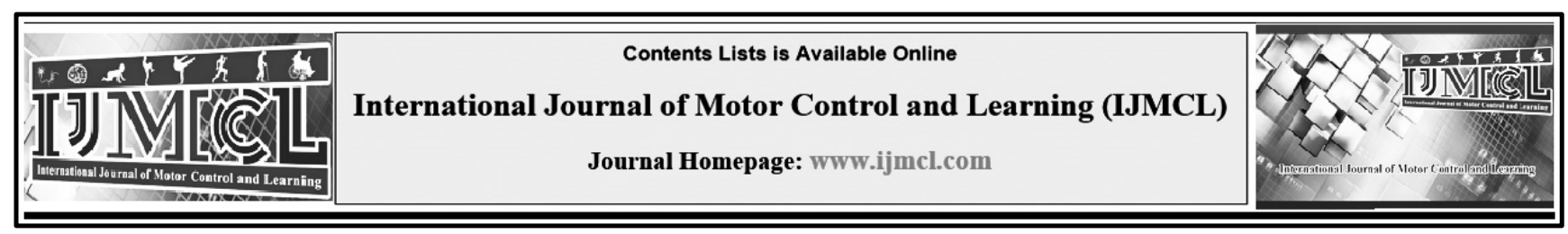

\title{
Effect of Resistance Training on Joint Position Sense and Disability in Adult Women with Multiple Sclerosis
}

\author{
Ashraf Asgari $^{a}$, Saeed Arsham ${ }^{b *}$, Afkham Daneshfar $^{c}$
}

${ }^{a}$ Master Degree in Motor Behavior, Kharazmi University, Tehran, Iran

${ }^{\mathrm{b}}$ Assistant Professor in Motor Behavior, Kharazmi University, Tehran, Iran

${ }^{\mathrm{c}}$ Associate Professor, Department of Motor Behavior, Faculty of Sport Sciences, Alzahra University, Tehran, Iran

\section{Keywords \\ Joint Position Sense \\ Multiple Sclerosis \\ Resistance Training}

Saeed Arsham,

Email: saeedarsham@khu.ac.ir

\begin{abstract}
Background: Multiple Sclerosis is a chronic disease of the central nervous system that is caused by destruction of myelin of the neuronal cells.

Objectives: This study aimed at determining the effect of resistance training on joint position sense in patients with multiple sclerosis.

Methods: This was a quasi-experimental study with the pretest-posttest design. Of 60 people referring to the National Multiple Sclerosis Association of Iran, 12 women aged 2055 years were selected based on physical disability scale developed by Kurtzke (1-4) and their fitness for participation in a sports protocol. They were randomly assigned to two groups: resistance training and control. The experimental group performed resistance training for 6 weeks, while the control group did not have any physical activity during this period. A goniometer was used to evaluate the joint position sense.

Results: The results of covariance analysis indicated a significant difference between the mean scores of two experimental and control groups joint position sense at an angle of 40 degrees $(\mathrm{P}<0.05)$. Resistance training at the starting knee flexion angle (40 degrees) led to progression of joint position sense, while it had no significant effect on the end angle (60 degrees). The effect of resistance training was also significant on physical disability $(\mathrm{P}=0.001)$. Six weeks of resistance training reduced reconstruction error of the 40-degree angle.

Conclusions: People with multiple sclerosis can improve the sensory aspect of movement, their physical ability, thereby the pattern and quality of their daily functional movements by these exercises. It is suggested that these exercises be used along with other rehabilitation programs to rehabilitate these patients.
\end{abstract}

\section{Introduction}

Multiple sclerosis (MS), which is most commonly diagnosed in women and during the ages of 20 and 40, is the most common progressive disease of the nervous system that by destroying myelin of nerves disrupts nerve and electrical conduction. Then, some muscles lose their capabilities (Soltani et al, 2009). Loss of myelin sheath leads to insulation deterioration in axons, decreases the conduction velocity of impulses, and eventually inhibits the transfer of action potentials, which is associated with decreasing isometric, isotonic, isokinetic force and total quadriceps muscle performance (Harrison, 1987). Due to the wide involvement of various levels of the neuronal axis, signs of physical disability such as muscle control difficulty and imbalance associated with walking instability, body inconsistency, tremor, dizziness, sudden movement of limbs and weakness, particularly in the legs, are observed (Mirshafiei, 2008). In addition to signs of physical 
disability, sensory symptoms, like numbness, loss of sensation, paresthesia, etc., are variable and common at the onset or in the early stages of MS recurrence, due to occurrence of spinal cord blood platelets in the posterior spinal column or in the brain stem (Greenwood et al. 2011). Sensory symptoms, as a kind of feedback from limbs to central nervous system, include a variety of components such as joint position sense, sense of speed and sense of force. In the meantime, joint position sense, that is, the ability to understand limb position in space without senses of vision and hearing under central and environmental mechanisms, also suffers (Neysi, Ebrahimi, \& Gohari, 2006). Although depth sense leads to the planning of musculoskeletal system, and thus dynamic joint stability, as Richie (2001) reported, a reduction in depth sense impulses of joint receptors can lead to abnormal body position and reduced reflex responses. Reduction of muscle strength, low endurance, and functional damages caused by decreased activity are the main disorders which limit daily activities of these patients and can be due to the illness itself or its long duration (White et al. 2004). Although the increase in strength does not change the course of the disease, the strength of muscle groups may prevent subsequent weakness resulting from non-usage of muscles (Barofsky, 2004). People with MS had long been banned from participating in physical activities because of the instability of symptoms during exercise; during the last decade, however, exercise activities have been recommended to these patients because of their beneficial effects (Motl et al. 2008). It has been shown that proper resistance exercises increase the muscle strength in multiple sclerosis (Barofsky, 2004; Khodadadeh et al. 2011). Improved strength of these patients is probably due to neuromuscular adaptations such as hypertrophy; the possible reason for this is the increased ability to coordinate the muscle group to perform a motion (Huisinga et al., 2011).

Through a study on patients with patellofemoral pain, Hazneci et al. (2005) concluded that isokinetic exercises with an angular velocity of 40 degrees per second and 180 degrees per second for six weeks improved inactive reconstruction of the knee joint position sense. However, Sekir et al. (2007) reported that ankle joint position sense was reduced after six weeks of isokinetic training for 10 degrees of inversion. Regardless of the different effect of different forms of exercise on improved depth sense, it is also important to consider how intervention effects or proper measurement angle of limb position sense are assessed. Accordingly, Neysi et al. (2006) examined the effect of starting angle and target angle on measuring knee joint depth sense in healthy men. When 10 degrees angles are selected, the mean absolute error is reduced and is more accurate when reconstructing different target angles compared to 90 degrees angle. Studying the effect of sensory training on position sense, speed and movement of elderly and young adults, Westlake et al. (2007) did not observe any progress in position sense and joint motion, except for improvement in differentiating the speed sense during passive movements. Pointing out the fact 
that joint position sense during passive movement is not sensitive enough to age-related changes, they noted that it is necessary to consider the same physiological factors, including strength or proper resistance interventions. Meanwhile, Peixoto et al. (2011) did not find any significant correlation between muscle function and joint position sense, as well as age and strength, while controlling age and gender of the subjects. According to them, muscle strength is more effective on physical function than depth sense; moreover, the isokinetic test may not well determine the nature of this relationship. Although the results of some older interventional studies showed that sport exercises or strength tests had no effect on joint position sense (for example, Stillman et al. 1998), it may be necessary to study the contradiction in results of other studies such as Thompson et al (2003) considering the nature of exercise, the type of muscle contraction and characteristics of the subject. They showed that resistance training had a significant effect on improving joint position sense of female elderly housewives. Considering the predictive factors of balance as an index of physical ability in people with MS, Fry et al. (2016) highlighted the limb joint position sense through a regression model and claimed that functional strength is indirectly related to balance, while sensory aspects of movement are the most involved.

Jamali et al. (2017) examined the relationship between sensory disorders and balance and quality of life of people with MS; it was found that the lower extremity depth sense plays a major role in their functional ability and it was concluded that exercise interventions, particularly resistance activities, may improve depth sense and improve the balance and reduce the dangers of falling in these patients.

Although numerous studies on MS patients have reported similar results in some variables such as motor function, balance or quality of life or fatigue associated with psychological factors and physical disability, there are some contradictions about the effects of exercise on improved depth sense with respect to different variables such as age, gender or health status; there has been no study on effect of resistance training on improved depth sense, particularly on joint position sense and disability of people with MS. Also, according to literature, resistance training interventions can help improve joint and limb sense which is required for some functional activities by enhancing strength at the exercised motor angles (Sekir et al. 2007; Tripp et al. 2007). Moreover, regarding defects in a variety of medical treatments for MS patients, it is reasonable to seek interventional training methods so that disabilities of patients can be reduced with minimal complications. In addition, the low level of activity observed in these patients plays an important role in daily activities of these people such that in a study on the effect of a sports program on 54 patients with MS, exercise has been shown to improve aerobic fitness, strength, and mobility of these patients (Schapira, 2000). Accordingly, this study tends to determine whether resistance training is effective in joint position sense response and physical disability of people 
with MS. Findings of this study help to understand the role of resistance exercises along with other therapeutic and rehabilitation interventions for rehabilitation of MS patients.

\section{Method}

This was an applied quasi-experimental study with a pretest-posttest design. Of 60 women with MS referring to the National Multiple Sclerosis Association of Iran, 12 women aged 20-55 years were selected based on results of exercise pretests (joint position sense and muscle strength) and their fitness for participation in the sports protocol. Then, they were randomly assigned to control and resistance training groups. The inability of these patients was considered in the range 1 to 4 , based on the Kurtzke's expanded disability status scale (EDSS); for this purpose, the patients were examined by a neurologist .

Individual data including age, marital status, educational level, height, and weight, as well as clinical data including disease history, MS type, disease duration, the frequency of recurrences, the number of hospitalization due to MS, and the latest EDSS score were recorded .

Kurtzke's expanded disability status scale (EDSS): this scale was used to evaluate disability of patients. EDSS was developed by Kurtzke. This scale measures different states and functions of the central nervous system, including pyramidal pathway, cerebrospinal, brain stem, sensory, visceral, visual functions, as well as brain system function. On the EDSS scale, the patient earns a score of 0 to 10 depending on the severity of the disease; higher EDSS score indicates higher disability (Kurtzke, 1983). A manual goniometer was also used to measure joint position sense.

Muscular strength assessment: manual muscle test (MMT) was used to measure the strength of quadriceps muscle in kilograms. It uses a handheld device which actually measures the quantitative value of the extroverted muscle power. Using this tool, the peak force required to break the isometric contraction is measured when the examiner applies a force opposite to the subject. Cuthbert and Goodheart (2007) verified the formal validity, content validity and construct validity of the MMT test and reported a high coefficient of reliability for it.

\section{Procedure}

Pre-resistance exercise stage: In the first stage, before resistance training begins, knee joint position sense was measured in both groups using angular reconstruction and goniometer. For this purpose, 4 red circular markers (diameter of $4 \mathrm{~cm}$ ) were attached to the outside of the tested limb on four points. This device is laid in the outer part of the thigh and leg parallel to a line which connects large trochanter of the hip joint at the top, the external Epicondyle in the middle, and the external ankle at the bottom (Lafortune et al. 1993). Accordingly, the subject was asked to sit on an adjustable seat which was placed next to a calibrated wall. The starting position was from 90 degrees of flexion to 40 and 60 -degree flexion. The examiner passively took the blindfolded subject's leg to 40 degrees and the subjects actively kept the 


\section{Original article}

IJMCL 2019; 1(1) 2-12

leg at the same degree for 5 seconds. Then, the examiner passively took the subject's leg to the starting position; the subject reconstructed this angle in 5 attempts, each for 3 seconds. The angle of each attempt was marked on the wall. Total variability was calculated. To prevent fatigue, the subject rested 5 seconds. This was repeated for the 60 degrees angle (Ribeiro et al, 2007).

Quadriceps muscle strength was evaluated using MMT test six days after the knee joint position sense was measured. The subject was asked to sit on an adjustable chair next to a calibrated wall. The starting position was from 90 degrees of flexion to the 40 and 60 degrees of flexion. The middle of the subject's leg was marked and the MMT power meter was placed in this part. The subject's leg was taken to the 40 degrees angle. The examiner applied pressure on the device for 3 seconds; the subject's strength was recorded in $\mathrm{Nm} / \mathrm{kg}$. The subject made four attempts at this angle, one practice attempt and three attempts with maximum power. Between each repetition, 15 seconds of rest was considered (Palmer \& Epler, 1990). This was repeated for the 60-degree angle. The average and maximum torque of all three attempts were calculated and normalized to the body weight of each subject. This value was obtained by dividing the torque by body weight in kilogram:

$\underline{\text { Quadrilateral muscle strength (Newton) } \times \text { Leg length }}$ Body Weight (Kilogram)

\section{Resistance training session}

After two training sessions, six weeks (three times a week) resistance exercises began. After 5 to 10 minutes of warm-up (walking and stretching), the participants performed resistance exercises for 25 to 30 minutes. To do this, the weights weighing one percent of their body weight was attached to them; every 2 weeks, 1 to 1.5 percent of the body weight was added to the weights. The movements used included getting up from the chair, lunge, the stairs move, lifting the legs and bending knees, which were done in three sets, each in 10 iterations. Participants cooled their body for 5-10 minutes. To prevent fatigue and increase body temperature, 30 seconds to 1-minute rest was considered between movements (Dalgas et al. 2008). After six weeks of resistance training, knee joint position sense and quadriceps muscle strength of both groups were remeasured by goniometer and MMT, respectively.

\section{Statistical method}

After determining the mean and standard deviation of data, Shapiro-Wilk test was used to check the assumption of normal distribution of variables; Leven test was used to test the homogeneity of variance of errors. Covariance analysis was used to compare the post-test scores using SPSS 18 software $(\mathrm{P}<0.05)$.

\section{Results}

Descriptive characteristics of subjects including mean height, weight, and EDSS are presented in Table 1. 
Table 1: Mean (standard deviation) of demographic variables of subjects.

\begin{tabular}{ccc}
\hline Variables & Control Group & Experimental Group \\
\hline Age (year) & $44.33(3.44)$ & $46.64(3.92)$ \\
\hline Height (Centimeter) & $165.50(5.68)$ & $165.50(6.15)$ \\
\hline Weight (kilogram) & $71.33(5.71)$ & $69.83(8.47)$ \\
\hline EDSS & $2.76(0.612)$ & $2.68(0.549)$ \\
\hline
\end{tabular}

Table 2: Mean (standard deviation) of Strength, Sense of Joint Position and EDSS Scores.

\begin{tabular}{cccc}
\hline Variable & Test Phase & Control Group & Experimental Group \\
\hline \multirow{2}{*}{ Strength at angle 40 } & Pretest & $5.35(0.976)$ & $5.08(1.18)$ \\
\cline { 2 - 4 } & Posttest & $4.69(1.22)$ & $5.93(1.03)$ \\
\hline \multirow{2}{*}{ Strength at angle 60 } & Pretest & $5.04(0.895)$ & $4.47(1.11)$ \\
\cline { 2 - 4 } & Posttest & $4.47(0.895)$ & $5.62(1.07)$ \\
\hline \multirow{2}{*}{ Joint position at angle 40 } & Pretest & $56.33(5.07)$ & $51.40(11.64)$ \\
\cline { 2 - 4 } Joint position at angle 60 & Posttest & $58.46(4.57)$ & $50.20(6.41)$ \\
\cline { 2 - 4 } & Pretest & $67.30(4.14)$ & $65.93(5.27)$ \\
\hline \multirow{2}{*}{ EDSS } & Posttest & $68.06(4.89)$ & $64.03(3.96)$ \\
\hline & Pretest & $2.76(0.612)$ & $2.68(0.549)$ \\
\hline & Posttest & $2.58(0.668)$ & $2.46(0.488)$ \\
\hline
\end{tabular}

Table 3: Shapiro-Wilk Test Results for the Natural Distribution of Data.

\begin{tabular}{ccc}
\hline Variables & Shapiro-Wilk & Significance Level \\
\hline Strength at angle 40 & 0.872 & 0.070 \\
\hline Strength at angle 60 & 0.879 & 0.085 \\
\hline Joint position at angle 40 & 0.883 & 0.095 \\
\hline Joint position at angle 60 & 0.939 & 0.458 \\
\hline EDSS & 0.902 & 0.170 \\
\hline Weight & 0.925 & 0.352 \\
\hline
\end{tabular}

Table 4: Leven Test for Homogeneity Analysis of Variance of Dependent Variables Errors.

\begin{tabular}{ccccc}
\hline Variables & $\begin{array}{c}\text { Leven F- } \\
\text { statistic }\end{array}$ & $\begin{array}{c}\text { Degree of Freedom } \\
\mathbf{1}\end{array}$ & $\begin{array}{c}\text { Degree of Freedom } \\
\mathbf{2}\end{array}$ & $\begin{array}{c}\text { Significance } \\
\text { Level }\end{array}$ \\
\hline Strength at angle 40 & 3.05 & 1 & 10 & 0.111 \\
\hline Strength at angle 60 & 1.156 & 1 & 10 & 0.308 \\
\hline $\begin{array}{c}\text { Joint position at angle } \\
\mathbf{4 0}\end{array}$ & 0.959 & 1 & 10 & 0.351 \\
\hline $\begin{array}{c}\text { Joint position at angle } \\
\mathbf{6 0}\end{array}$ & 0.014 & 1 & 10 & 0.907 \\
\hline EDSS & 1.48 & 1 & 10 & 0.251 \\
\hline
\end{tabular}


As shown in Table 3 and Table 4, p-value $>0.05$ indicates normal distribution of data and homogeneous variance of errors.

Controlling the effect of the pretest, Table 5 shows a significant difference between strength of two groups at $40^{\circ}$ angle $(\mathrm{F}=16.27, \mathrm{P}<0.001)$ and strength of two groups at $60^{\circ}$ angle
$(\mathrm{F}=19.01, \quad \mathrm{P}<0.001)$. Therefore, it can be concluded that resistance training is effective (0.64) on 40 and $60^{\circ}$ strength of adult women with MS. Test power is 0.947 for $40^{\circ}$ angle and 0.971 for $60^{\circ}$.

Table 5: Results of Covariance Analysis of Groups for Sensing the Joint Position at 40- and 60-Degree Angles.

\begin{tabular}{cccccccc}
\hline Sources of Change & Sum of Squares & df & Average of Squares & F & P & $\begin{array}{c}\text { Eta } \\
\text { Square }\end{array}$ \\
\hline \multirow{4}{*}{40 Degree } & Pretest & 2.325 & 1 & 2.325 & 0.068 & 0.800 & 0.068 \\
\cline { 2 - 8 } & Group & 176.147 & 1 & 176.147 & 5.149 & 0.048 & 0.364 \\
\cline { 2 - 9 } & Error & 307.889 & 9 & 34.210 & & & \\
\cline { 2 - 9 } & Total & 35940.560 & 11 & & & & \\
\hline \multirow{3}{*}{$\mathbf{6 0}$ Degree } & Pretest & 0.004 & 1 & 0.004 & 0.000 & 0.990 & 0.000 \\
\cline { 2 - 9 } & Group & 47.754 & 1 & 47.754 & 2.16 & 0.175 & 0.194 \\
\cline { 2 - 9 } & Error & 198.323 & 9 & 22.036 & & & \\
\cline { 2 - 9 } & Total & 52598.360 & 11 & & & & \\
\hline
\end{tabular}

Table 6: Results of Covariance Analysis of Groups for EDSS.

\begin{tabular}{ccccccc}
\hline Sources of Change & Sum of Squares & df & Average of Squares & F & P & Eta Square \\
\hline Pretest & 3.332 & 1 & 3.332 & 310.801 & 0.001 & 0.972 \\
\hline Group & 0.296 & 1 & 0.296 & 25.135 & 0.001 & 0.736 \\
\hline Error & 0.096 & 9 & 0.011 & & & \\
\hline Total & 88.670 & 11 & & & & \\
\hline
\end{tabular}

Table 5 shows that controlling the effect of the pretest will make a significant difference between joint position sense of two groups at $40^{\circ}$ angle in posttest $(\mathrm{F}=5.14, \mathrm{P}<0.005)$, while there is no significant difference between joint position sense of two groups at $60^{\circ}$ angle $(\mathrm{F}=2.16, \quad \mathrm{P}>0.005)$. Therefore, it can be concluded that resistance training is effective (0.36) on $40^{\circ}$ joint position sense of adult women with MS. Test power is 0.526 . However, resistance training is not effective on $60^{\circ}$ joint position sense of adult women with MS.
Table 6 shows a significant difference between EDSS of two groups in posttest $(\mathrm{F}=25.135$, $\mathrm{P}<0.001)$. Therefore, it can be concluded that resistance training is effective (0.73) on EDSS of adult women with MS. Test power is 0.993 .

\section{Discussion and Conclusion}

This study tended to determine the effect of resistance training on joint position sense and disability in adult women with multiple sclerosis (MS).

Findings of this study showed that resistance training had a significant effect on joint position sense of adult women with MS at angle 40, but it 
had no significant effect on angle 60. Meeuwsen et al. (1993) investigated the effect of change in accuracy of ankle joint position sense as a result of repetition on young and elderly people and concluded that exercise was effective in improving joint position sense. The probable reason for this consistency can be attributed to active angular reconstruction in both studies. Refshauge et al. (1995) also found that resistance training was effective in improving joint position sense by examining ankle joint position sense in weight bearing activities versus other non-weight bearing activities. Weight-bearing activities can probably be considered as a kind of weight training exercise, although the amount of load imposed in the present study is lower and it is a low percentage of total weight. Petrall et al. (1997) divided people based on their physical activity over the past year that did not focus on resistance exercises; their age (19-68) was also considered as an effective variable on joint position sense. Despite their age classification, it seems that regular exercise can maintain or improve depth sense accuracy by increasing the tensile strength of muscular duct (Goodman \& Marks, 1998); this is consistent with current study. This has been confirmed by Bouët and Gahéry (2000). They pointed out that active exercise with voluntary limb and muscular involvement could be effective in improving joint position sense, where resistance exercises here also yield results consistent with this idea. Other authors (e.g .Thompson, 2003; Hazneci, 2005; and Sekir, 2007) have also pointed to positive effects of exercises on joint position sense. Although both
Hazneci (2005) and Sekir (2007) used isokinetic interventions in similar training sessions (six weeks), it seems that passive reconstruction in these types of exercises had results similar to the active reconstruction of joint movement in exercises of this study (isotonic).

Baker et al. (2002) reported that the mean absolute error was significantly higher in a sitting position at $60^{\circ}$ angle than $40^{\circ}$ angle, which is consistent with the current study. As these authors claimed, limb position or the test can affect muscle contraction or joint loading pattern (Bartlett, 2007); thus, consistency of results can be attributed to the similarity of two positions. Another explanation is that, despite the controversy over the major task of joint receptors, some researchers believe that capsular-ligament receptors play a more important role in the extreme angles of motion, in contrast to muscle receptors which are effective in determining knee position sense in all domains (Shumway-Cook \& Woollacott 2012). The resistance training of the present study led to the progression of joint position sense in the starting knee flexion angle; however, its effect was not significant at the end angles .

On the other hand, Stillman et al. (1998) reported that muscle resistance activities did not have a significant effect on joint position sense. This is inconsistent with the current study. The inconsistency can be attributed to the fact that they used inactive motion reconstruction to evaluate depth sense. As Edin (2001) pointed out, it is muscle duct that is responsible for providing motion-related information in passive movements, 
not joint receptors. In addition, their subjects were athletes, and this may have led to less influence and adaptation of resistance activities compared to normal or disabled people (Dalgas et al. 2008).

Another finding contradictory with the results related to joint position sense was the study done by Westlake et al. (2007) on the elderly, pointing to other factors, including age and strength. For a better explanation of potentially effective factors, this study considered the promoted strength of subjects; clearly, as Table 5 shows, strength significantly increased in both angles. Supporting Thompson et al (2003), the increased muscle strength is probably an effective factor in improving joint position sense.

Another finding of this study was that resistance training was effective on EDSS score of adult women with MS and reduced physical disability score. Only two very close studies to this study reported inconsistent results. First, White et al (2004) examined the effect of eight months of resistance training on MS patients; although the score of disability was reduced and it was improved, the improvement was not significant. According to them, various factors together such as style or duration of exercise and fatigue control may lead to this result. Second, Romberg et al (2004) studied MS patients after 26 weeks of combined exercise and reported no significant improvement in physical disability. They not only observed any difference in knee muscle strength of control and experiment groups, but also considered self-report of people on doing prescribed activities as their limitations.
Consistent with the current study, DeBolt and McCubbin (2004) and Dodd et al. (2006) claimed that a significant reduction in disability of people with MS is associated with improved strength, particularly in the lower extremity. After eight weeks of water exercise interventions for people with MS, Smith et al. (2009) and Salem et al. (2011) achieved similar results and reported that physical disability of patients was significantly reduced. It is likely that limb movement in water has played a role in resistance training; however, one should not ignore other factors related to their exercise, including the reduced fatigue, pain, or mental aspects which may affect disability scores.

Because lower strength in people with MS is due to metabolic disorders of muscle, muscle atrophy due to inactivity and also due to neural tube obstruction and lower development of muscular tension (Pérez et al. 2007), resistance training has been able to improve neurological adaptations, including the use of motor units and synchronization of neuronal motor contraction and lead to optimal motor performance and reduction of disability scores. This was previously approved by Gutierrez et al. (2005).

The results of other researchers, including Brett (2011), Peixoto et al. (2011), Fry et al. (2016) and Jamali et al. (2017) were consistent with the current study in improving the disability due to exercise interventions. Regardless of the methodology, they attributed the improvement in disability of these patients to factors such as increased balance and strength or reduced fatigue and even psychosocial aspects. Future studies should address some 
limitations of the current study. Fortunately, or unfortunately, the sample size was small, which can be effective in generalizing the results to the considered population. Considering proper intervals to reduce fatigue of subjects, its value was not objectively measured. Finally, exercise interventions on patients with higher disabilities as well as follow-up studies may clarify the effects of activity on general function and their quality of life.

\section{Acknowledgement}

The authors appreciate the authorities in the MS Society of Iran and participants in this study.

\section{References}

1. Soltani, M., Hejazi, S. M., Noorian, A., Zendedel, A., \& Ashkanifar, F. (2009). The effect of aerobic training on the improvement of expanded disability status scale (EDSS) in multiple sclerosis patients. J Mashhad School Nurs Midw; 9(2): 107-13.

2. Salami, F (1995). Neurological diseases, in addition to the major manifestations of neurological diseases. Tehran: Hayyan Publications.

3. Mirshafiyi, S. A (2008). Multiple Sclerosis. Tehran: Hayyan Publications.

4. Greenwood, N. L., Duffell, L. D., Alexander, C. M., \& McGregor, A. H. (2011). Electromyographic activity of pelvic and lower limb muscles during postural tasks in people with benign joint hypermobility syndrome and non-hypermobile people. A pilot study. Manual therapy, 16(6), 623-628.

5. Neisi, K., Ebrahimi, A., Goharpey, S. (2005). Investigating the effect of starting angle and target angle on measuring the sense of position of knee joint in healthy men. Jondishapur Scientific Medical Journal, No. 30.

6. Richie, D. H. (2001). Functional instability of the ankle and the role of neuromuscular control: a comprehensive review. The journal of foot and ankle surgery, 40(4), 240-251.

7. White, L. J., McCoy, S. C., Castellano, V., Gutierrez, G., Stevens, J. E., Walter, G. A., \& Vandenborne, K. (2004). Resistance training improves strength and functional capacity in persons with multiple sclerosis. Multiple Sclerosis Journal, 10(6), 668-674.

8. Barofsky, I. (2004). Quality of life research: A critical introduction. Quality of Life Research, 13(5), 10211024.
9. Motl, R. W., Arnett, P. A., Smith, M. M., Barwick, F. H., Ahlstrom, B., \& Stover, E. J. (2008). Worsening of symptoms is associated with lower physical activity levels in individuals with multiple sclerosis. Multiple Sclerosis Journal, 14(1), 140-142.

10. Khodadadeh, S., Kordi, M., Khosravi, N., Sanglaji, B. (2010). Effect of 8 weeks of selected exercise on walking distance, strength and fatigue of MS patients. Journal of Hayatno Rehabilitation Clinic. Volume 10, Issue 2, pp. 28-22.

11. Huisinga, J. M., Filipi, M., \& Stergiou, N. (2011). Elliptical exercise improves fatigue ratings and quality of life in patients with multiple sclerosis. Journal of Rehabilitation Research \& Development. 48, 881- 890.

12. Hazneci, B., Yildiz, Y., Sekir, U., Aydin, T., \& Kalyon, T. A. (2005). Efficacy of isokinetic exercise on joint position sense and muscle strength in patellofemoral pain syndrome. American journal of physical medicine \& rehabilitation, 84(7), 521-527.

13. Sekir, U., Yildiz, Y., Hazneci, B., Ors, F., \& Aydin, T. (2007). Effect of isokinetic training on strength, functionality and proprioception in athletes with functional ankle instability. Knee surgery, sports traumatology, arthroscopy, 15(5), 654-664.

14. Westlake, K. P., Wu, Y., \& Culham, E. G. (2007). Sensory-specific balance training in older adults: effect on position, movement, and velocity sense at the ankle. Physical therapy, 87(5), 560-568.

15. Peixoto, J. G., Dias, J. M. D., Dias, R. C., da Fonseca, S. T., \& Teixeira-Salmela, L. F. (2011). Relationships between measures of muscular performance, proprioceptive acuity, and aging in elderly women with knee osteoarthritis. Archives of gerontology and geriatrics, 53(2), 253-257.

16. Stillman, B. C., McMeeken, J. M., \& Macdonell, R. A. (1998). Aftereffects of resisted muscle contractions on the accuracy of joint position sense in elite male athletes. Archives of physical medicine and rehabilitation, 79(10), 1250-1254.

17. Thompson, K. R., Mikesky, A., Bahamonde, R. E., \& Burr, D. B. (2003). Effects of physical training on proprioception in older women. $\mathrm{J}$ Musculoskelet Neuronal Interact, 3(3): 223-231.

18. Fry, D. K., Huang, M. H., \& Rodda, B. J. (2016). Predictors of static balance in ambulatory persons with multiple sclerosis. International Journal of Rehabilitation Research, 39(1), 42-47.

19. Jamali, A., Sadeghi, E., \& Fereshtenejad, N. (2015, May). Sensory Impairment and Its Relationship with Balance and Quality Of Life in People with Multiple Sclerosis. In Multiple Sclerosis Journal (Vol. 21, No. 6, pp. 813-813).

20. Tripp, B. L., Yochem, E. M., \& Uhl, T. L. (2007). Recovery of upper extremity sensorimotor system acuity in baseball athletes after a throwing-fatigue protocol. Journal of athletic training, 42(4), 452.

21. Schapira, RT. (2000). Symptom management and rehabilitation. In Burks JS Johnson KP. Multiple Sclerosis, Diagnosis, Management and Rehabilitation. New York. Demos Medical Publishing, 86:315-320. 
22. Kurtzke, J. F. (1983). Rating neurologic impairment in multiple sclerosis: an expanded disability status scale (EDSS). Neurology, 33(11), 1444-1452.

23. www.lafayetteinstrument.com

24. Cuthbert, S. C., \& Goodheart, G. J. (2007). On the reliability and validity of manual muscle testing: a literature review. Chiropractic \& osteopathy, 15(1), 4.

25. Lafortune, M. A., Lambert, C. E., \& Lake, M. J. (1993). Skin marker displacement at the knee joint. Journal of biomechanics, 26(3), 299.

26. Ribeiro, F., Mota, J., \& Oliveira, J. (2007). Effect of exercise-induced fatigue on position sense of the knee in the elderly. European journal of applied physiology, 99(4), 379-385.

27. Palmer, M. L., \& Epler, M. (1990). Principles of examination techniques. Clinical assessment procedures in physical therapy. Philadelphia: JB Lippincott, 8-36.

28. Dalgas, U., Stenager, E., \& Ingemann-Hansen, T. (2008). Multiple sclerosis and physical exercise: recommendations for the application of resistance, endurance and combined training. Multiple Sclerosis Journal, 14(1), 35-53.

29. Meeuwsen, H. J., Sawicki, T. M., \& Stelmach, G. E. (1993). Improved foot position sense as a result of repetitions in older adults. Journal of gerontology, 48(3), 137-141.

30. Refshauge, K. M., \& Fitzpatrick, R. C. (1995). Perception of movement at the human ankle: effects of leg position. The Journal of physiology, 488(1), 243248.

31. Petrella, R. J., Lattanzio, P. J., \& Nelson, M. G. (1997). Effect of age and activity on knee joint proprioception. American Journal of Physical Medicine \& Rehabilitation, 76(3), 235-241. 50.

32. Goodman, M., \& Marks, R. (1998). The association between knee proprioception and isotonic quadriceps femoris strength. Physiotherapy Canada, 50(1), 53-57.

33. Bouët, V., \& Gahéry, Y. (2000). Muscular exercise improves knee position sense in humans. Neuroscience letters, 289(2), 143-146.

34. Baker, V., Bennell, K., Stillman, B., Cowan, S., \& Crossley, K. (2002). Abnormal knee joint position sense in individuals with patellofemoral pain syndrome. Journal of Orthopaedic Research, 20(2), 208214.

35. Arsham, S. (2011) Introduction to Sport Biomechanics. Islamic Azad University, Dezful Branch.

36. Shumway-Cook. A., Woollacott M.H. (2012). Motor Control (Translating Research into Clinical Practice). Publisher: Lippincott Williams \& Wilkins.

37. Edin, B. B. (2001). Cutaneous afferents provide information about knee joint movements in humans. The Journal of physiology, 531(1), 289-297.

38. Romberg, A., Virtanen, A., Ruutiainen, J., Aunola, S., Karppi, S. L., Vaara, M. \& Seppänen, A. (2004). Effects of a 6-month exercise program on patients with multiple sclerosis: A randomized study. Neurology, 63(11), 2034-2038.
39. DeBolt, L. S., \& McCubbin, J. A. (2004). The effects of home-based resistance exercise on balance, power, and mobility in adults with multiple sclerosis. Archives of physical medicine and rehabilitation, 85(2), 290-297.

40. Dodd, K. J., Taylor, N. F., Denisenko, S., \& Prasad, D. (2006). A qualitative analysis of a progressive resistance exercise programe for people with multiple sclerosis. Disability and rehabilitation, 28(18), 11271134.

41. Smith, C., Hale, L., Olson, K., \& Schneiders, A. G. (2009). How does exercise influence fatigue in people with multiple sclerosis?. Disability and rehabilitation, 31(9), 685-692.

42. Salem, Y., Scott, A. H., Karpatkin, H., Concert, G., Haller, L., Kaminsky, E. \& Spatz, E. (2011). Community-based group aquatic program for individuals with multiple sclerosis: a pilot study. Disability and rehabilitation, 33(9), 720-728.

43. Pérez, C. A., Sánchez, V. M., Teixeira, F. D. S., \& Fernández, J. D. P. (2007). Effects of a resistance training program in multiple sclerosis Spanish patients: a pilot study. Journal of sport rehabilitation, 16(2), 143153.

44. Gutierrez, G. M., Chow, J. W., Tillman, M. D., McCoy, S. C., Castellano, V., \& White, L. J. (2005). Resistance training improves gait kinematics in persons with multiple sclerosis. Archives of physical medicine and rehabilitation, 86(9), 1824-1829.

45. Brett, S. (2011). Balance exercises for people with multiple sclerosis. Nursing standard, 15(33), 47-52. 57. 